\title{
5 TaskForce Tarmed
}

Die Gesamtrevision des Tarmed-Tarifs kommt langsam, aber sicher in die Schlussphase. Mitte August 2015 soll das Kapitel Ultraschall eingereicht werden können. Im Jahr 2016 soll sich der Bundesrat dazu äußern. Die Taxpunktwert-Verhandlungen werden voraussichtlich kantonal durchgeführt. Wo wir dann schlussendlich landen werden, ist noch ziemlich ungewiss...

Beat Dubs, Leiter TaskForce Tarmed 